\title{
Correlation between tumor microenvironment-associated factors and the efficacy and prognosis of neoadjuvant therapy for rectal cancer
}

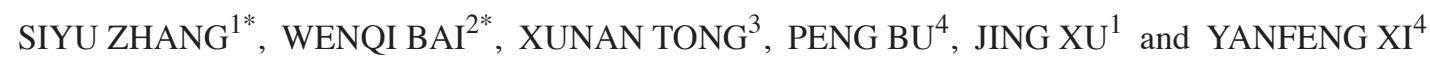 \\ ${ }^{1}$ Department of Pathology, Shanxi Medical University, Taiyuan, Shanxi 030001; ${ }^{2}$ Department of Colorectal Surgery, \\ Shanxi Provincial Cancer Hospital, Taiyuan, Shanxi 030013; ${ }^{3}$ Departent of Surgery, Shanxi Medical University, \\ Taiyuan, Shanxi 030001; ${ }^{4}$ Department of Pathology, Shanxi Provincial Cancer Hospital, Taiyuan, Shanxi 030013, P.R. China
}

Received March 4, 2018; Accepted September 20, 2018

DOI: $10.3892 / \mathrm{ol} .2018 .9682$

\begin{abstract}
The tumor microenvironment contributes to the survival and development of tumor cells and is therefore a key target for cancer therapy. The tumor microenvironment has unique physical and chemical properties and is associated with inflammation and immunity. To examine the correlation between tumor microenvironment-associated factors and the efficacy and prognosis of neoadjuvant therapy for rectal cancer, and to compare the differences between two treatments [neoadjuvant chemotherapy (NAC) vs. neoadjuvant chemoradiotherapy (NACR)], an immunohistochemical method was used to measure the expression levels of $\mathrm{CD}^{+}$ tumor-infiltrating lymphocytes (TILs), cluster of differentiation (CD) $8^{+}$TILs, forkhead box P3 (FOXP3) ${ }^{+}$TILs, cytotoxic T lymphocyte-associated antigen- $4^{+}$TILs and programmed death ligand-1 (PD-L1) ${ }^{+}$TILs in 109 patients with rectal cancer, preand post-neoadjuvant therapy. The significance of these protein expression patterns was also analyzed using tissue microarrays, and the prognostic significance of these findings evaluated. The results indicated that high levels of CD4 ${ }^{+}$TILs, CD8 ${ }^{+}$TILs and PD-L1+TILs may be associated with favorable responses to neoadjuvant therapy, whereas high levels of FOXP ${ }^{+}$TILs were associated with poor therapeutic responses. Expression levels of CD8 ${ }^{+}$TILs and FOXP3 ${ }^{+}$TILs following neoadjuvant therapy were independent prognostic factors and affected the total survival of patients subjected to neoadjuvant therapy for
\end{abstract}

Correspondence to: Professor Yanfeng $\mathrm{Xi}$, Department of Pathology, Shanxi Provincial Cancer Hospital, 3 Workers' New Village, Xinghualing, Taiyuan, Shanxi 030013, P.R. China

E-mail: xiyanfeng1998@163.com

Professor Jing Xu, Department of Pathology, Shanxi Medical University, 56 Xinjian South Road, Taiyuan, Shanxi 030001, P.R. China

E-mail: xujing@sxmu.edu.cn

*Contributed equally

Key words: rectal cancer, tumor microenvironment, neoadjuvant therapy, tumor-infiltrating lymphocytes, tissue microarrays the treatment of rectal cancer. Moreover, the effects of NAC and NACR on the tumor microenvironment may be different.

\section{Introduction}

Rectal cancer is a common malignant tumor of the digestive tract (1). The recurrence and mortality rates of rectal cancer are high due to the unique anatomy of the rectum. The standard treatment for locally advanced rectal cancer is preoperative concurrent chemoradiotherapy or short-range radiotherapy+total mesorectal excision+postoperative adjuvant chemotherapy. Neoadjuvant therapy may improve the anus preservation rate and reduce the risk of tumor recurrence. However, the efficacy of neoadjuvant therapy is variable and little is known about the factors associated with therapeutic efficacy. The disadvantages of therapy failure include delayed surgery and immunosuppression. The tumor microenvironment comprises immunological cells with local infiltration of cancer stromal cells together with their secreted active mediators and tumor cells. In the 1880 s, Paget (2) established the concept of 'seed and soil'. As a 'soil,' the tumor microenvironment provides the basis for tumor occurrence, development, invasion and metastasis (3). Tumor-infiltrating lymphocytes (TILs) are an important component of this microenvironment and serve a vital role in tumor progression and treatment outcome. However, alterations in the expression levels of TILs in the tumor microenvironment, pre- and post-neoadjuvant therapy, are not fully understood.

Cluster of differentiation (CD) $4^{+} \mathrm{T}$ and $\mathrm{CD} 8^{+} \mathrm{T}$ serve a crucial role in tumor recognition and removal. $\mathrm{CD}^{+}{ }^{+} \mathrm{T}$ cells kill tumor cells through interferon- $\gamma(\mathrm{IFN}-\gamma)$ and activate $\mathrm{CD} 8^{+} \mathrm{T}$ cells in various ways. Activated $\mathrm{CD}^{+} \mathrm{T}$ cells are recruited to the tumor site and induce apoptosis (4). Regulatory T cells (Tregs) are a subgroup of $\mathrm{T}$ cells that inhibit the immune response to autologous tumor cells, and this inhibition is considered the main cause of the failure of immunotherapy (5-8). The transcription factor forkhead box P3 (Foxp3) is considered to be the most specific Treg marker. Tregs may inhibit $\mathrm{T}$ cells by expressing cytotoxic $\mathrm{T}$ lymphocyte-associated antigen-4 (CTLA-4), which binds to B7 molecules on the surface of activated $\mathrm{T}$ cells. It also reduces the activation of $\mathrm{T}$ cells and degrades activated $\mathrm{T}$ cells by combining 
with CD80 and CD86 on the surface of antigen presenting cells, which converts transduction signals, producing indolamine 2,3-dioxygenase and degrading tryptophan (9). Previous studies have reported that Foxp3 may be associated with poor prognosis; however, its role in the prognosis of rectal cancer is controversial.In addition, the activation of $\mathrm{T}$ cells requires the concomitant release of secondary signals by costimulators $(10,11)$. CTLA-4 inhibits the activation of T cells by interacting with B7 (CD80/CD86) (12), and previous studies have reported that CTLA-4 is associated with poor prognosis $(13,14)$. Another inhibitor located on the surface of T lymphocytes, B7-H1 (10), also known as programmed death ligand-1 (PD-L1), is expressed in T cells, B cells, macrophages and dendritic cells, and its expression is upregulated following the activation of antigen-presenting cells. PD-L1 may inhibit the proliferation of $\mathrm{T}$ cells and the production of cytokines in $\mathrm{T}$ cells by combining with PD-1, and thus serves a critical role in immune tolerance and escape $(15,16)$. It has been reported that PD-L1 is upregulated in numerous malignant tumors, including melanoma, lung cancer, renal cell carcinoma, ovarian cancer, colorectal cancer (17), breast cancer (18) and osteosarcoma (19), and may serve an important role in tumor-immune system interactions $(20,21)$.

In the present study, the clinical treatment and prognosis of rectal cancer was evaluated by selecting immune markers associated with tumor progression in TILs, including CD4, CD8, CTLA-4, Foxp3 and PD-L1. Alterations in the tumor microenvironment were assessed pre- and post-neoadjuvant therapy by immunohistochemistry (IHC), and the tumor microenvironment, curative effect and prognosis of rectal cancer were compared between neoadjuvant chemotherapy (NAC) and neoadjuvant chemoradiotherapy (NACR).

\section{Materials and methods}

Patients. The present study investigated 109 patients who underwent neoadjuvant therapy in the Shanxi Provincial Cancer Hospital (Shanxi, China) between January 2012 and December 2015, of whom 50 patients were treated using the FOLFOX4 regimen every 2-3 weeks (3 days of chemotherapy +2 weeks of rest) for two to four cycles of preoperative chemotherapy, and 59 patients were treated with chemoradiotherapy. In the latter group, the total dose of radiotherapy was 25-50 Gy, and two to four cycles of the FOLFOX4 regimen were provided during the same period. The Research Ethics Committee of the Shanxi Cancer Hospital approved the study and patient consent was obtained.

Tissue microarray. Tissue microarrays consisted of paraffin blocks in which 48 separate tissue cores were assembled in an array. The paraffin blocks were from 109 patients with rectal cancer who had undergone neoadjuvant treatment. A hollow needle was used to remove tissue cores as small as $1.8 \mathrm{~mm}$ in diameter from regions of interest in paraffin-embedded tissues. These tissue cores were inserted in a recipient paraffin block in a precisely spaced array. The tissue microarray block was placed upside down on the slide, incubated in an oven at $55^{\circ} \mathrm{C}$ for $10 \mathrm{~min}$, and cooled to room temperature. The tissue cores and the recipient paraffin fusion blocks were repeatedly produced. Sections from these blocks were cut using a microtome, mounted on a microscope slide, and analyzed using a microscope.
IHC. All biopsy specimens collected prior to treatment and resected specimens collected following treatment were analyzed using IHC. The paraffin sections $(3 \mu \mathrm{m})$ were dewaxed in xylene and hydrated in gradient ethanol solutions, and antigen retrieval was performed in a microwave for $2 \mathrm{~min}$. The tissue slides were incubated in 3\% hydrogen peroxide for $10 \mathrm{~min}$, and non-specific binding was blocked using normal goat serum (SP900, working solution; OriGene Technologies, Inc., Beijing, China) for 5-10 min at $25^{\circ} \mathrm{C}$. The slides were washed in PBS and incubated with the primary antibody at $4^{\circ} \mathrm{C}$ overnight. The slides were again washed in PBS, incubated with the secondary antibody (GK600705A, goat anti-mouse/rabbit IgG, multimer, working solution; GeneTech Biotechnology Co., Ltd.) for $30 \mathrm{~min}$ at $25^{\circ} \mathrm{C}$, and visualized for 5 min with a diaminobenzidine color reaction kit (GK347005; GeneTech Biotechnology Co., Ltd.) at $25^{\circ} \mathrm{C}$. The slides were counterstained with hematoxylin for $50 \mathrm{~s}$ at $25^{\circ} \mathrm{C}$, dehydrated and mounted following transparency. The primary antibodies were as follows: CD4 (cat. no. EP204; GeneTech Biotechnology Co., Ltd., Shanghai, China); CD8 (cat. no. C8/114B; GeneTech Biotechnology Co., Ltd.); CTLA-4 (cat. no. sc-376016; Santa Cruz Biotechnology, Inc., Dallas, TX, USA); FOXP3 (cat. no. 236A/E7; Abcam, USA); PD-L1 (cat. no. sp142; GeneTech Biotechnology Co., Ltd.). The positive controls were human tonsils for CTLA4 and FOXP3, and human placenta for PD-L1. An isotype control was used as a negative control for each case stained for CTLA-4, FOXP3 and PD-L1, to control for potential false positive staining. The microscope we use is OLYMPUS, BX46 (Olympus Corporation, Tokyo, Japan) at x40 magnification.

Histological analysis. The double-blind method was used for the interpretation of histological sections by two pathologists. The percentage and average number of positive TILs were calculated in five fields at $\mathrm{x} 40$ magnification. Tumor response was evaluated using the tumor regression grade (TRG) system proposed by Dworak et al (22) as follows: i) Grade 0, no regression; ii) grade 1 , minor tumor regression, dominant tumor mass with evident fibrosis in $£ 25 \%$ of the tumor mass; iii) grade 2, moderate tumor regression, with fibrosis in $26-50 \%$ of the tumor mass; iv) grade 3 , high tumor regression $(>50 \%)$, fibrosis in the majority of the tumor mass; and v) grade 4 , total tumor regression, absence of viable tumor cells, only fibrotic mass remaining. In the present study, TRGs 3 and 4 indicated a good response to therapy, whereas TRGs 0-2 indicated a poor response to therapy. The mean value was set as the cut-off value for the density of each type of TIL and patients were classified into high- and low-TIL groups based on this cut-off value.

Follow-up. From the initial diagnosis, all patients were followed-up until August 15th 2017, and the median follow-up period was 42 months. All patients were monitored by outpatient appointment or telephone follow-up. Overall survival (OS) was defined as the period from pathological diagnosis to mortality.

Statistical analysis. SPSS version 23.0 (IBM Corp., Armonk, NY, USA) was used for data analysis. The $\chi^{2}$ test was used to compare categorical data. Data are expressed as the mean \pm standard deviation. A t-test was used for group comparisons. Pearson correlation was used for correlation analysis. 
The Kaplan-Meier test was used for single-factor analysis of patient survival. The Cox proportional hazard regression model was used for multi-factor analysis of prognosis. $\mathrm{P}<0.05$ was considered to indicate a statistically significant difference.

\section{Results}

Patient characteristics. Among the 109 patients, 62 were male and 47 were female. The age of the study population was 32-78 years (mean, $54.78 \pm 10.71$ years). The pathological types included high differentiation (eight cases), moderate differentiation ( 76 cases), and low differentiation ( 25 cases). With respect to the depth of infiltration: 33 cases were in stage $\mathrm{T} 3$ and 76 cases were in stage T4. A total of 35 patients did not present lymph node metastasis, whereas 74 patients did present lymph node metastasis. Tumor distance from the anal margin was $0-14 \mathrm{~cm}$ (mean $5.02 \pm 2.55 \mathrm{~cm}$ ). Overall, 34 patients had a good response, whereas 75 patients presented a poor response (Table I).

Evaluation of immune markers. CD4 was expressed on the cell membrane of interstitial TILs (Fig. 1A). CD8 was expressed in the cytoplasm of interstitial TILs (Fig. 1B). CTLA4 and FOXP3 were expressed in the cytoplasm of TILs and tumor cells; positive cells were identified by staining (Fig. 1C and D). PD-L1 was expressed in the nucleus of TILs and tumor cells; positive cells were identified by staining (Fig. 1E). The percentage of positive cells in CD4 ${ }^{+}$TILs pre- and post-neoadjuvant therapy was $16.35 \pm 8.76$ and $19.95 \pm 8.73 \%$, respectively $(\mathrm{P}=0.002)$. The percentage of positive cells in CD $8{ }^{+}$TILs pre- and post-neoadjuvant therapy was $17.64 \pm 7.74$ and $21.27 \pm 10.21 \%$, respectively $(\mathrm{P}=0.001)$. The percentage of positive cells in CTLA-4 ${ }^{+}$TILs pre- and post-neoadjuvant therapy was $6.34 \pm 5.66$ and $8.88 \pm 7.17 \%$, respectively $(\mathrm{P}=0.003)$. There was no significant difference between the percentage of positive cells in FOXP3 ${ }^{+}$TILs pre- and post-neoadjuvant therapy $(\mathrm{P}=0.065)$. The percentage of positive cells in $\mathrm{PD}-\mathrm{L} 1^{+} \mathrm{TILs}$ preand post-neoadjuvant therapy was $9.28 \pm 8.77$ and $5.83 \pm 8.12 \%$, respectively ( $\mathrm{P}=0.001$; Fig. 2).

Association between immune markers, clinicopathological features and curative effect pre-and post-neoadjuvant therapy. Patients with poorly differentiated adenocarcinoma were more likely to have a good response to neoadjuvant therapy ( $\mathrm{P}=0.007$; Table II). Prior to neoadjuvant therapy, patients with low FOXP3 ${ }^{+}$TILs, and high PD-L1 ${ }^{+}$TILs were more likely to have a favorable therapeutic response $(\mathrm{P}<0.001$ and $\mathrm{P}=0.001$, respectively). Following neoadjuvant therapy, patients with high CD $4^{+}$TILs, high CD8 ${ }^{+}$TILs, low FOXP $3^{+}$TILs and high PD-L1'TILs were more likely to have a good response to therapy $(\mathrm{P}=0.026,0.007,0.007$ and $<0.001$, respectively). The other clinicopathological features and tumor immune markers were not significantly associated with the curative effect.

Survival analysis. Single-factor analysis of the total survival time was conducted using the Kaplan-Meier test and indicated the presence of an association between the total survival period, lymphatic invasion, CD8 ${ }^{+}$TILs and FOXP $3^{+}$TILs following neoadjuvant therapy. The survival function curve demonstrated that the total survival time of patients without lymph node metastasis was long and the mean survival time was
Table I. Clinicopathological characteristics of the 109 patients.

Clinicopathological parameters

No. patients $(n=109)$

Sex

Male

$62(57 \%)$

Female

$47(43 \%)$

Age, mean (SD)

$54.78(10.71)$

$\leq 55$

$57(52 \%)$

$>55$

$52(48 \%)$

Histology

Low

$8(7 \%)$

Middle

$76(70 \%)$

High

$25(23 \%)$

T stage

T3

$33(30 \%)$

$\mathrm{T} 4$

$76(70 \%)$

Lymphatic invasion

Negative

$35(32 \%)$

Positive

$74(68 \%)$

DFTAV, cm [mean (SD)]

$5.02(2.55)$

$\leq 5$

$69(63 \%)$

$>5$

$40(37 \%)$

TRG

Poor response

$75(69 \%)$

Good response

$34(31 \%)$

Survival rate

Survival

$89(82 \%)$

Mortality

$20(18 \%)$

DFTAV, distance from the anal verge; TRG, tumor regression grade; SD, standard deviation.

$25.73 \pm 3.93$ months $(P=0.038$; Fig. $3 \mathrm{~A})$. Total survival time in patients with high levels of CD8 ${ }^{+}$TILs was long, and the average survival time was $26.25 \pm 2.15$ months $(\mathrm{P}=0.032$; Fig. $3 \mathrm{~B})$. The total survival time in patients with low FOXP3 ${ }^{+}$TIL levels was long and the mean survival time was $25.06 \pm 2.53$ months $(\mathrm{P}=0.016$; Fig. $3 \mathrm{C})$. The median survival time of patients whose CD4 TILs did not increase following neoadjuvant therapy was 19 months. The median survival time of patients whose CD8 ${ }^{+}$TILs did not increase following neoadjuvant therapy was 21.5 months. Multiple factor analysis with the Cox proportional risk regression model was used to analyze the single factors that displayed statistical significance. Multiple stepwise regression analysis indicated that CD8 ${ }^{+}$TILs and FOXP3 ${ }^{+}$TILs were independent prognostic factors and may affect the total survival of patients subjected to neoadjuvant therapy for rectal cancer ( $\mathrm{P}=0.037$ and 0.013 , respectively; Table III).

Correlation between immune markers. There was no significant correlation between CD $4^{+}$TIL levels with CTLA- $4^{+}$TIL and FOXP3 ${ }^{+}$TIL levels following neoadjuvant therapy (P-values of 0.398 and 0.186 , respectively). There was no correlation between CD8 ${ }^{+}$TIL levels with CTLA- $4^{+}{ }^{T I L}$ and FOXP3 ${ }^{+}$TIL 
A

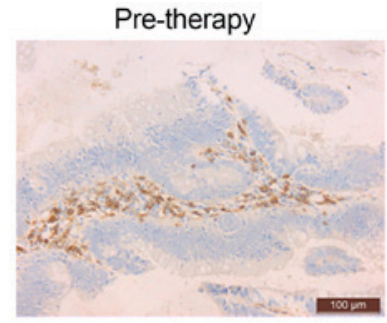

B

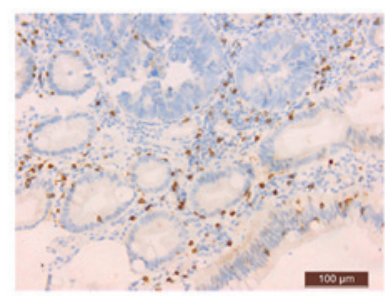

C

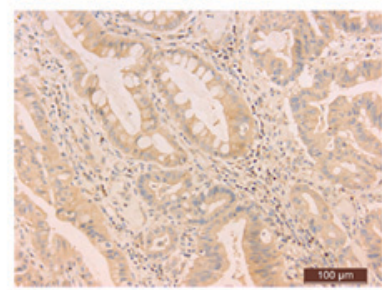

D

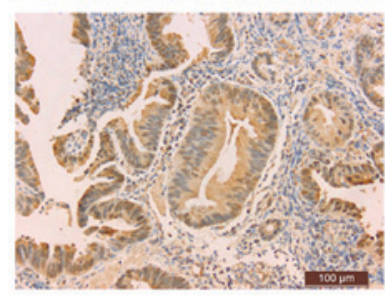

E

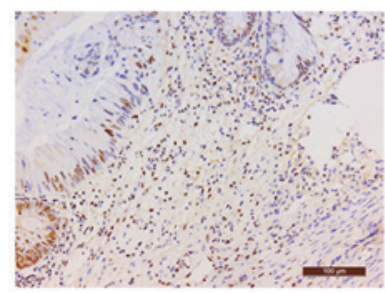

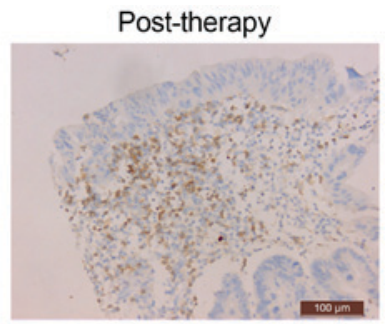
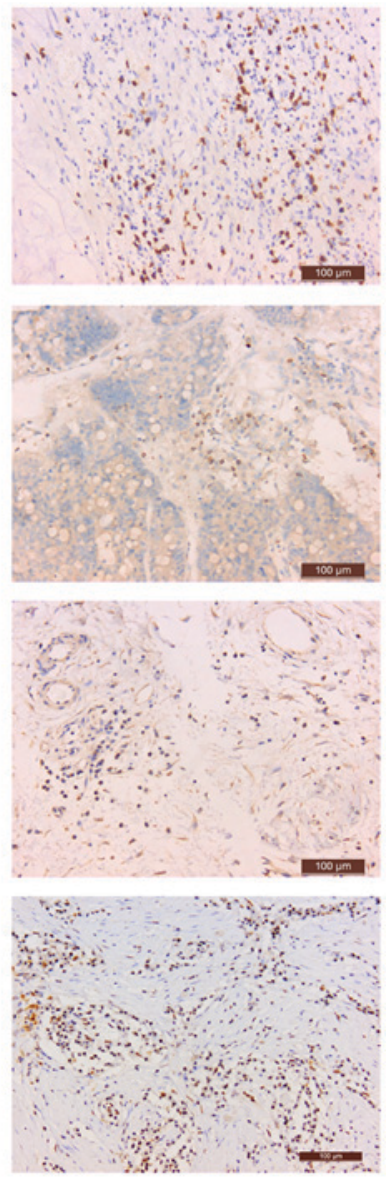

Figure 1. Positive expression of tumor microenvironment-associated factors in rectal cancer pre- and post-neoadjuvant therapy, determined by immunohistochemistry. Magnification, x200. (A) CD4+TILs. (B) CD8 ${ }^{+}$TILs (C) CTLA-4+TILs. (D) FOXP3+TILs. (E) PD-L1+TILs. CD, cluster of differentiation; TILs, tumor infiltrating lymphocytes; PD-L1, programmed death ligand-1; FOXP3, forkhead box P3.

levels following neoadjuvant therapy $(\mathrm{P}=0.845$ and 0.655 , respectively) (Data not shown). However, there was a strong correlation between CTLA- $4^{+}$TIL levels and FOXP3 ${ }^{+}$TIL levels (Pearson correlation coefficient $=0.252, \mathrm{P}=0.008$; Fig. 4).

Comparison between NAC and NACR. The comparison of immune markers, survival time, curative effect and survival rate between NAC and NACR indicated that the percentage of CTLA-4 ${ }^{+}$TILs-positive cells was significantly higher in NACR compared with NAC $(\mathrm{P}<0.001)$. The differences in other indexes were not statistically significant (Table IV).

\section{Discussion}

The results of the present study indicated that the expression of CD4 $4^{+}$TILs and CD8 ${ }^{+}$TILs was significantly higher

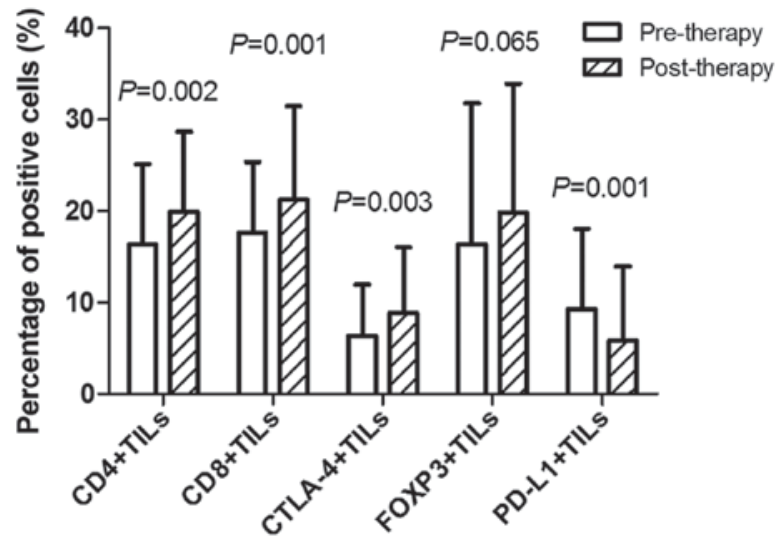

Figure 2. Comparison of the expression levels of different immune parameters pre- and post-neoadjuvant therapy. CD, cluster of differentiation; TILs, tumor infiltrating lymphocytes; PD-L1, programmed death ligand-1; FOXP3, forkhead box P3; CTLA, cytotoxic T lymphocyte-associated antigen.

post-neoadjuvant therapy compared with pre-neoadjuvant therapy, and patients with high levels of CD4 ${ }^{+}$TILs and CD8 ${ }^{+}$TILs following neoadjuvant therapy were more responsive to neoadjuvant therapy. This result demonstrated that these patients may have had immunodeficiencies prior to neoadjuvant therapy, and radiotherapy and chemotherapy may increase the expression levels of CD $4^{+}$TILs and CD $8^{+}$TILs. A previous study has reported that chemotherapy may induce the expression of death receptors, increase the levels of CD4 $4^{+}$TILs, CD8 ${ }^{+}$TILs and other immune cells, and activate the immune microenvironment of the tumor (23), which may improve the response to neoadjuvant therapy. However, there was no significant difference in the effect of CD4 ${ }^{+} \mathrm{TIL}$ and $\mathrm{CD} 8^{+} \mathrm{TIL}$ levels prior to neoadjuvant therapy on its curative effect, indicating that the effect of chemotherapy and/or radiotherapy on the tumor immune microenvironment may be more important in neoadjuvant therapy compared with the pre-existing local immune response. Following neoadjuvant therapy, prognosis in patients with high CD8 ${ }^{+}$TIL levels was significantly better compared with patients with low CD8 ${ }^{+}$TIL levels, suggesting that the levels of CD8 ${ }^{+}$TILs in the tumor microenvironment may limit the occurrence and development of tumors and improve prognosis in patients with high $\mathrm{CD} 8^{+} \mathrm{TILs}$. This result is consistent with those of other studies $(24,25)$.

The CTLA- 4 costimulatory pathway is a negative signal activated by $\mathrm{T}$ cells. In the present study, the expression level of CTLA-4 ${ }^{+}$TILs post-neoadjuvant therapy was significantly higher compared with pre-neoadjuvant therapy. This result may be due to the killing effect of chemotherapy or chemoradiotherapy in the tumor, leading to the release of vascular endothelial growth factor, interleukin-10 (IL-10), transforming growth factor- $\beta$ and other inhibitory factors by tumor cells (26). Moreover, the expression of major histocompatibility complex molecules or costimulatory molecules CD80/86 on the surface of dendritic cells, and CTLA-4 molecules as CD80/86 receptors alters accordingly (26). However, there was no significant correlation between CTLA-4 ${ }^{+}$TILs levels and the effect of neoadjuvant therapy pre- and post-neoadjuvant therapy.

Compared with levels prior to neoadjuvant therapy, the number of FOXP3+TILs was not significantly increased following neoadjuvant therapy. However, low numbers of 
Table II. Association between immune markers, clinicopathological features, and curative effect pre- and post-neoadjuvant therapy.

Clinicopathological features

Sex

Male

Female

Age, years

Histology

Low

Middle

High

T stage

T3

T4

Lymphatic invasion

Negative

Positive

Distance from the anal verge, $\mathrm{cm}$

$\leq 5$
$>5$

Pre-therapy CD4+TILs, (\%)

Low

High

Post-therapy CD4+TILs, (\%)

Low

High

Pre-therapy CD8+TILs (\%)

Low

High

Post-therapy CD8+TILs (\%)

Low

High

Pre-therapy CTLA-4+TILs (\%)

Low

High

Post-therapy CTLA-4+TILs (\%)

Low

High

Pre-therapy FOXP3+TILs (\%)

Low

High

Post-therapy FOXP3 ${ }^{+}$TILs (\%)

Low

High

Pre-therapy PD-L1 ${ }^{+}$TILs (\%)

Low

High

Post-therapy PD-L1+TILs (\%)

Low

High

\section{Poor response}

43

32

$55.87 \pm 10.94$

11

57

7

25

50

21

54

44

31

45

30

48

27

47

28

45

30

48

27

44

31

36

39

39

36

52

23

62

13

10
Good response

$\mathrm{P}$-value

0.887

19

15

$52.38 \pm 9.89$

0.116

14

19

1

8

26

14

20

25

9

14

20

14

20

16

0.126

18
11

23

21

0.823

13

26

8

30

$<0.001^{\mathrm{a}}$

4

27

7

$0.007^{\mathrm{a}}$

12

$0.001^{\mathrm{a}}$

22

14

20

$0.007^{\mathrm{a}}$

0.072

${ }^{a} \mathrm{P}<0.05$. CD, cluster of differentiation; TILs, tumor infiltrating lymphocytes; PD-L1, programmed death ligand-1; FOXP3, forkhead box P3; CTLA, cytotoxic T lymphocyte-associated antigen. 

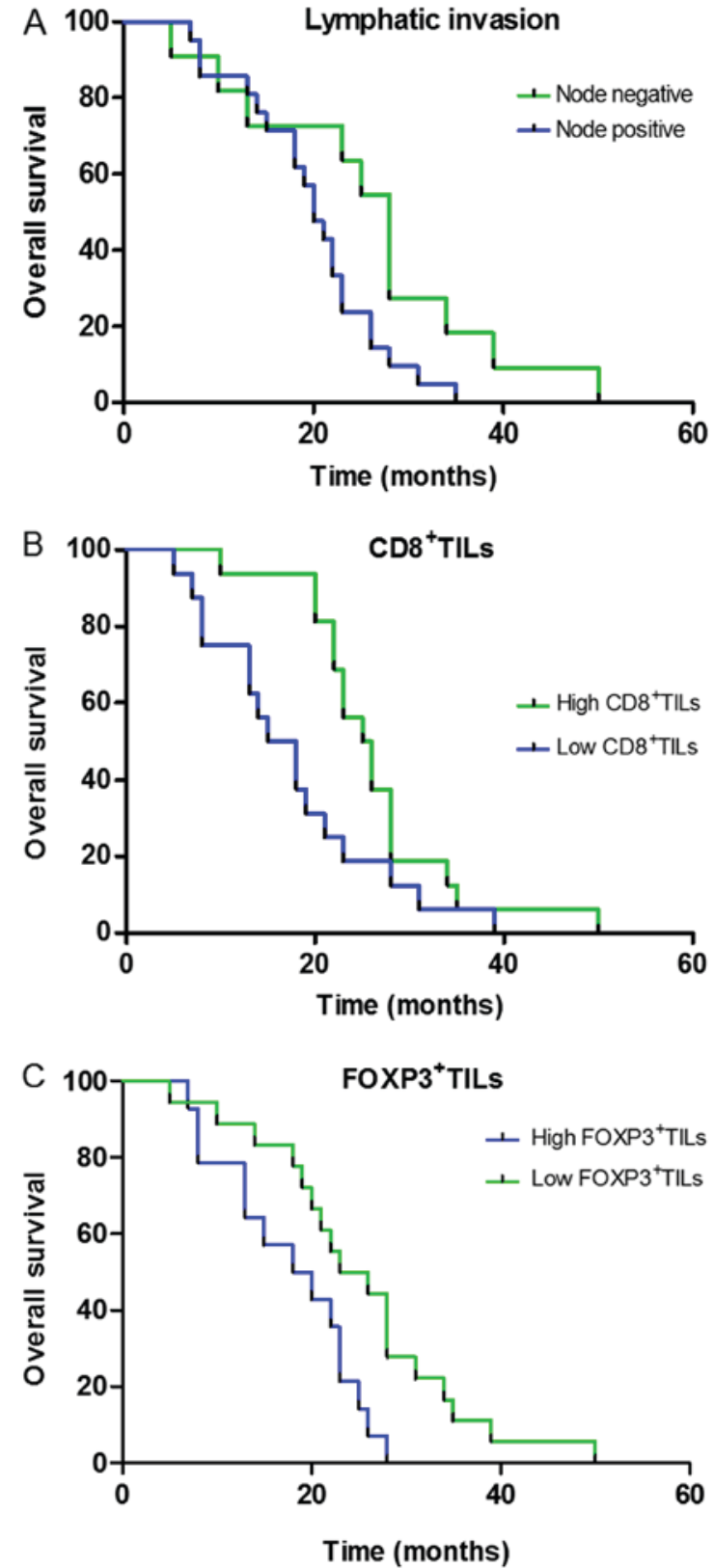

Figure 3. Single-factor analysis survival curves. Kaplan-Meier estimates for OS. (A) A comparison of the OS rates between node-negative and node-positive patients. An absence of lymphatic invasion was significantly associated with increased OS $(\mathrm{P}=0.038)$. (B) A comparison of the OS rates between high and low expression levels of CD8 ${ }^{+}$TILs. High CD8 ${ }^{+}$TIL expression levels were significantly associated with increased $\mathrm{OS}(\mathrm{P}=0.032)$. (C) A comparison of the OS rates between high and low expression levels of FOXP3'TILs. Low FOXP ${ }^{+}$TIL repression levels were significantly associated with increased OS $(\mathrm{P}=0.016)$. OS, overall survival; $\mathrm{CD}$, cluster of differentiation; TILs, tumor-infiltrating lymphocytes; FOXP3, forkhead box P3.

FOXP3+TILs were more effective pre- and post-neoadjuvant therapy. Tregs undergo rapid turnover compared with other $\mathrm{T}$ cell subsets and are selectively depleted by a number of chemotherapeutic drugs, including 5-fluorouracil (27-29). In numerous patients, selective depletion by chemotherapy may enhance the 'window of opportunity' for anti-tumor immunity and promote tumor regression. However, the possibility that the number of adverse reactions is increased due to Tregs in the neoplasm following neoadjuvant therapy cannot be excluded. The results of the present study indicate that the prognosis of

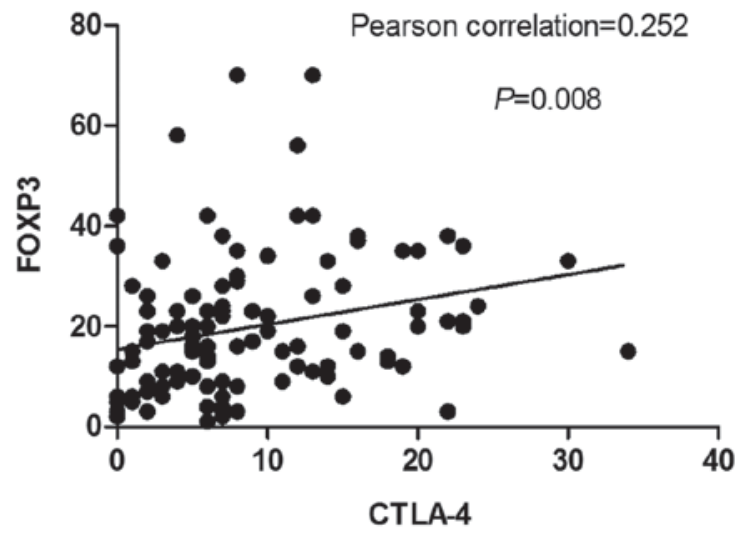

Figure 4. A positive correlation was observed between CTLA-4+TIL expression levels and FOXP3+TIL expression levels. CTLA, cytotoxic T lymphocyte-associated antigen; FOXP3, forkhead box P3.

patients with low expression levels of FOXP3+TILs following neoadjuvant therapy is improved. However, the association between FOXP ${ }^{+}{ }^{+}$TILs levels and the occurrence and development of neoplasms remains unclear. Frey et al (30) demonstrated that the high expression level of FOXP3+ TILs is associated with good prognosis, whereas Lin et al (31) reported that levels of FOXP $3^{+}$TILs are associated with tumor progression. Therefore, the correlation between FOXP3+TILs and tumor progression requires further investigation.

Anti PD-1 and anti PD-L1 antibodies have been demonstrated to have a notable and lasting effect in patients with melanoma, renal cell carcinoma and non-small cell lung cancer (31). However, clinical trials have demonstrated that the blocking effect of anti PD-1 or PD-L1 antibodies in rectal cancer is poor (32). In the present study, the percentage of positive PD-L1+TILs post-neoadjuvant therapy was lower compared with pre-neoadjuvant therapy. However, the effect of chemotherapy on the expression levels of PD-L1 is unknown. Saigusa et al (33) used quantitative polymerase chain reaction analysis to measure alterations in the expression levels of the $P D-L 1$ gene in four types of colon cancer cell lines, prior to irradiation and 1,3 , and 5 days following irradiation. It was observed that the expression levels of $P D-L 1$ were reduced following irradiation. However, the mechanism underlying this process requires further investigation. Patients with high expression levels of PD-L1+TILs pre-and post-neoadjuvant therapy may achieve a good therapeutic response, which contradicts the results of a previous study (33). The PD-1/PD-Ll signaling pathway may serve a negative role in regulating $\mathrm{T}$ cells through multiple mechanisms, although PD-1 is not the only receptor mediating PD-L1 activity. PD-L1 also causes apoptosis of PD-1-negative T lymphocytes, suggesting that there may be additional receptors expressed on $\mathrm{T}$ lymphocytes that bind to PD-L1 and induce immunosuppression (34). Moreover, in contrast with its immunosuppressive activity, PD-L1 may also stimulate the immune response. PD-L1 protein in combination with low levels of anti-CD3 antibody in resting $\mathrm{T}$ cells may enhance the proliferation of $\mathrm{T}$ cells as well as the secretion of IL-10 and IFN $\gamma$-1b (35). These contradictory results suggest that PD-L1 may have multiple receptors, which exert different immunomodulatory effects through binding with different receptors (36). 
Table III. Correlation between clinicopathological features, immune markers and total survival time.

\begin{tabular}{|c|c|c|c|c|c|}
\hline \multirow[b]{2}{*}{ Clinicopathological features } & \multicolumn{2}{|c|}{ Single factor analysis } & \multicolumn{2}{|c|}{ Multifactor analysis } & \multirow[b]{2}{*}{ P-value } \\
\hline & Log-rank & P-value & B & SD & \\
\hline Gender & 0.764 & 0.382 & & & \\
\hline Age & 0.360 & 0.548 & & & \\
\hline T staging & 0.016 & 0.898 & & & \\
\hline Lymphatic invasion & 4.313 & $0.038^{\mathrm{a}}$ & 0.455 & 0.424 & 0.063 \\
\hline DFTAV, cm & 0.486 & 0.486 & & & \\
\hline Pre-therapy CD4+TILs, $\%$ & 0.759 & 0.383 & & & \\
\hline Post-therapy CD $4^{+}$TILs, \% & 0.144 & 0.704 & & & \\
\hline Pre-therapy CD8 ${ }^{+}$TILs, $\%$ & 0.399 & 0.528 & & & \\
\hline Post-therapy CD8 ${ }^{+}$TILs, $\%$ & 4.680 & $0.032^{\mathrm{a}}$ & 2.191 & 0.377 & $0.037^{\mathrm{a}}$ \\
\hline Pre-therapy CTLA-4+TILs, \% & 0.090 & 0.764 & & & \\
\hline Post-therapy CTLA-4+TILs, \% & 2.831 & 0.092 & & & \\
\hline Pre-therapy FOXP3 ${ }^{+}$TILs, $\%$ & 0.630 & 0.427 & & & \\
\hline Post-therapy FOXP3+TILs, \% & 5.826 & $0.016^{\mathrm{a}}$ & 0.357 & 0.417 & $0.013^{\mathrm{a}}$ \\
\hline Pre-therapy PD-L1+TILs, $\%$ & 0.007 & 0.935 & & & \\
\hline Post-therapy PD-L1+TILs, \% & 2.267 & 0.132 & & & \\
\hline
\end{tabular}

${ }^{a} \mathrm{P}<0.05$. DFTAV, distance from the anal verge; $\mathrm{CD}$, cluster of differentiation; TILs, tumor infiltrating lymphocytes; PD-L1, programmed death ligand-1; FOXP3, forkhead box P3; CTLA, cytotoxic T lymphocyte-associated antigen; SD, standard deviation; B, beta.

Table IV. Comparison between NAC and NACR.

\begin{tabular}{|c|c|c|c|}
\hline Clinicopathological features & NAC & NACR & P-value \\
\hline \multicolumn{4}{|l|}{ Post-therapy CD4+TILs (\%) } \\
\hline Low & 29 & 33 & \multirow[t]{2}{*}{0.828} \\
\hline High & 21 & 26 & \\
\hline \multicolumn{4}{|l|}{ Post-therapy CD8 ${ }^{+}$TILs (\%) } \\
\hline Low & 26 & 30 & \multirow[t]{2}{*}{0.905} \\
\hline High & 24 & 29 & \\
\hline \multicolumn{4}{|l|}{ Post-therapy CTLA-4+TILs (\%) } \\
\hline Low & 41 & 29 & \multirow[t]{2}{*}{$<0.001^{\mathrm{a}}$} \\
\hline High & 9 & 30 & \\
\hline \multicolumn{4}{|l|}{ Post-therapy FOXP3+TILs (\%) } \\
\hline Low & 28 & 38 & \multirow[t]{2}{*}{0.371} \\
\hline High & 22 & 21 & \\
\hline \multicolumn{4}{|l|}{ Post-therapy PD-L1+TILs (\%) } \\
\hline Low & 38 & 38 & \multirow[t]{2}{*}{0.189} \\
\hline High & 12 & 21 & \\
\hline Survival time, months & $19.31 \pm 5.99$ & $22.92 \pm 10.98$ & 0.294 \\
\hline \multicolumn{4}{|l|}{ TRG } \\
\hline Poor response & 37 & 38 & \multirow[t]{2}{*}{0.281} \\
\hline Good response & 13 & 21 & \\
\hline Survival rate & 80 & 85 & 0.931 \\
\hline Survival & 41 & 48 & \\
\hline Mortality & 9 & 11 & \\
\hline
\end{tabular}

a $\mathrm{P}<0.05$. NAC, neoadjuvant chemotherapy; NACR, neoadjuvant chemoradiotherapy; $\mathrm{CD}$, cluster of differentiation; TILs, tumor infiltrating lymphocytes; PD-L1, programmed death ligand-1; FOXP3, forkhead box P3; CTLA, cytotoxic T lymphocyte-associated antigen; TRG, tumor regression grade. 
A previous study (37) reported that CTLA-4 and FOXP3 may inhibit the activation of $\mathrm{T}$ cells, however the results indicated no statistically significant association between the expression levels of CD4 ${ }^{+}$TILs or CD8 ${ }^{+}$TILs and CTLA-4+TILs or FOXP3+TILs following neoadjuvant therapy. This may be due to the small sample size. Nonetheless, there was a positive association between CTLA-4+TILs and FOXP3+TILs following neoadjuvant therapy. Tregs may inhibit the activity of effector cells by transducing a reverse signal of the crosslink between CTLA-4 and antigen-presenting cell or B7 (CD80 and CD86) on the surface of activated $\mathrm{T}$ cells (38). In addition, it was reported that the expression level of CTLA-4 on the surface of CD4+D25+Treg was decreased following knockdown of FOXP3 expression in mice, yet was increased in mice transfected with high levels of FOXP3 (39). The comparison between NAC and NACR indicated that the percentage of CTLA-4+TILs-positive cells in NACR was significantly higher compared with NAC. It is possible that the body produces an immunosuppressive response following radiation exposure. The expression of CTLA-4 may be beneficial for avoiding or reducing the occurrence of autoimmune reactions caused by radiation exposure. Radiation has been reported to activate anti-tumor immune responses by killing cancer cells and inducing distant effects (40). In addition, studies have demonstrated that radiation may increase the proportion of Treg cells in humans $(41,42)$, and Tregs may induce the expression of CTLA-4 through various pathways. Moreover, the present study observed a positive correlation between CTLA-4 ${ }^{+}$TIL levels and FOXP3+TIL levels, which also supports this hypothesis. In addition, it was reported that NACR was more effective and increased the OS compared with NAC; however, the difference was not statistically significant, which may be due to the small sample size.

Compared with previous studies, the number of patients in the current study was notably larger; the majority of studies currently in the literature were conducted with $<100$ patients $(33,43,44)$. In the analysis, the differences between the two methods of NAC and NACR were compared, and it was reported that the effects of these two treatments on numerous immune factors differed. However, the association between these indicators and the curative effect or prognosis with different treatment methods was not analyzed. There may be a number of differences in the results due to differences in the criteria for evaluating the pathological response and the standard of interpretation of IHC.

In conclusion, CD4+TILs and CD8 ${ }^{+}$TILs may inhibit the growth of tumor cells, and FOXP3 $3^{+}$TILs are associated with a poor response to therapy. CD8+TILs and FOXP3+TILs following neoadjuvant therapy are independent prognostic factors and affect total survival in patients undergoing neoadjuvant therapy for rectal cancer. The role of PD-L1+TILs remains to be investigated. The effects of NAC and NACR on the tumor microenvironment may be different. The tumor microenvironment is complex, thus in-depth studies on immunoregulatory mechanisms in the inflammatory microenvironment of rectal cancer cells, and a possible association between the tumor microenvironment and signal transduction and metabolic pathways of rectal cancer may elucidate the pathological mechanisms and potential immunotherapy of rectal cancer.

\section{Acknowledgements}

The authors would like to thank Ms. Wenxia Yan for her assistance with IHC; Mr. Liwu Xie for his assistance with data collection; and Dr Xiaojuan Wang and Dr Jing Li (Department of Pathology of Shanxi Provincial Cancer Hospital, Taiyuan, China) for their assistance with the image analysis and pathological assessment.

\section{Funding}

This study was funded by the Major Research and Development Project of Shanxi Science and Technology Department of Shanxi (grant no. 201603 D321049) and the Scientific Research Tsak of the Shanxi Health and Family Planning Commission of Shanxi (grant no. 2014052).

\section{Availability of data and materials}

The datasets used and/or analyzed during the current study are available from the corresponding author on reasonable request.

\section{Authors' contributions}

SYZ, WQB and YFX were responsible for the study design, original article drafting and editing, data acquisition and data analysis. XNT was responsible for original article drafting, data analysis and article revision. PB was responsible for the immunohistochemistry. JX and YFX performed the immunohistochemical evaluations and critically revised the manuscript.

\section{Ethics approval and consent to participate}

Ethics for the use of human tissues was approved by the Shanxi Provincial Cancer Hospital Ethics Committee (no. 201722) and patient consent was obtained.

\section{Patient consent for publication}

Not applicable.

\section{Competing interests}

The authors declare that they have no competing interests.

\section{References}

1. Torre LA, Bray F, Siegel RL, Ferlay J, Lortet-Tieulent J and Jemal A: Global cancer statistics, 2012. CA Cancer J Clin 65: 87-108, 2015 .

2. Paget S: The distribution of secondary growths in cancer of the breast. 1889. Cancer Metastasis Rev 8: 98-101, 1989.

3. Albini A, Magnani E and Noonan DM: The tumor microenvironment: Biology of a complex cellular and tissue society. Q J Nucl Med Mol Imaging 54: 244-248, 2010.

4. Kumamoto Y, Mattei LM, Sellers S, Payne GW and Iwasaki A: CD4+ $\mathrm{T}$ cells support cytotoxic $\mathrm{T}$ lymphocyte priming by controlling lymph node input. Proc Natl Acad Sci USA 108: 8749-8754, 2011.

5. Hori S, Nomura T and Sakaguchi S: Control of regulatory T cell development by the transcription factor Foxp3. Science 299: 1057-1061, 2003.

6. Grossman WJ, Verbsky JW, Barchet W, Colonna M, Atkinson JP and Ley TJ: Human T regulatory cells can use the perforin pathway to cause autologous target cell death. Immunity 21 : 589-601, 2004 
7. Paust S, Lu L, McCarty $\mathrm{N}$ and Cantor $\mathrm{H}$ : Engagement of B7 on effector $\mathrm{T}$ cells by regulatory $\mathrm{T}$ cells prevents autoimmune disease. Proc Natl Acad Sci USA 101: 10398-10403, 2004.

8. von Boehmer H: Mechanisms of suppression by suppressor T cells. Nat Immunol 6: 338-344, 2005.

9. Chaudhary B, Khaled YS, Ammori BJ and Elkord E: Neuropilin 1: Function and therapeutic potential in cancer. Cancer Immunol Immunother 63: 81-99, 2014.

10. Bloch O, Crane CA, Kaur R, Safaee M, Rutkowski MJ and Parsa AT: Gliomas promote immunosuppression through induction of B7-H1 expression in tumor-associated macrophages. Clin Cancer Res 19: 3165-3175, 2013.

11. Lu B, Chen L, Liu L, Zhu Y, Wu C, Jiang J and Zhang X: T-cell-mediated tumor immune surveillance and expression of B7 co-inhibitory molecules in cancers of the upper gastrointestinal tract. Immunol Res 50: 269-275, 2011.

12. Brunner MC, Chambers CA, Chan FK, Hanke J, Winoto A and Allison JP: CTLA-4-Mediated inhibition of early events of T cell proliferation. J Immunol 162: 5813-5820, 1999.

13. Salvi S, Fontana V, Boccardo S, Merlo DF, Margallo E, Laurent S, Morabito A, Rijavec E, Dal Bello MG, Mora M, et al: Evaluation of CTLA-4 expression and relevance as a novel prognostic factor in patients with non-small cell lung cancer. Cancer Immunol Immunother 61: 1463-1472, 2012.

14. Karabon L, Markiewicz M, Kosmaczewska A, Partyka A, Pawlak-Adamska E, Tomkiewicz A, Ciszak L, Jagoda K, Dzierzak-Mietla M, Kyrcz-Krzemien S and Frydecka I: Pretransplant donor and recipient CTLA-4 mRNA and protein levels as a prognostic marker for aGvHD in allogeneic hematopoietic stem cell transplantation. Immunol Lett 165: 52-59, 2015.

15. Zou W and Chen L: Inhibitory B7-family molecules in the tumour microenvironment. Nat Rev Immunol 8: 467-477, 2008.

16. Keir ME, Butte MJ, Freeman GJ and Sharpe AH: PD-1 and its ligands in tolerance and immunity. Annu Rev Immunol 26: 677-704, 2008

17. Dong H, Strome SE, Salomao DR, Tamura H, Hirano F, Flies DB, Roche PC, Lu J, Zhu G, Tamada K, et al: Tumor-associated B7-H1 promotes T-cell apoptosis: A potential mechanism of immune evasion. Nat Med 8: 793-800, 2002.

18. Sabatier R, Finetti P, Mamessier E, Adelaide J, Chaffanet M, Ali HR, Viens P, Caldas C, Birnbaum D and Bertucci F: Prognostic and predictive value of PDL1 expression in breast cancer. Oncotarget 6: 5449-5464, 2015.

19. Shen JK, Cote GM, Choy E, Yang P, Harmon D, Schwab J, Nielsen GP, Chebib I, Ferrone S, Wang X, et al: Programmed cell death ligand 1 expression in osteosarcoma. Cancer Immunol Res 2: 690-698, 2014

20. Curiel TJ, Wei S, Dong H, Alvarez X, Cheng P, Mottram P, Krzysiek R, Knutson KL, Daniel B, Zimmermann MC, et al: Blockade of B7-H1 improves myeloid dendritic cell-mediated antitumor immunity. Nat Med 9: 562-567, 2003.

21. Zhang L, Gajewski TF and Kline J: PD-1/PD-L1 interactions inhibit antitumor immune responses in a murine acute myeloid leukemia model. Blood 114: 1545-1552, 2009.

22. Dworak O, Keilholz L and Hoffmann A: Pathological features of rectal cancer after preoperative radiochemotherapy. Int J Colorectal Dis 12: 19-23, 1997.

23. Kalbasi A, June $\mathrm{CH}$, Haas $\mathrm{N}$ and Vapiwala $\mathrm{N}$ : Radiation and immunotherapy: A synergistic combination. J Clin Invest 123 2756-2763, 2013

24. Yasuda K, Nirei T, Sunami E, Nagawa H and Kitayama J: Density of CD4(+) and CD8(+) T lymphocytes in biopsy samples can be a predictor of pathological response to chemoradiotherapy (CRT) for rectal cancer. Radiat Oncol 6: 49, 2011.

25. Garcia-Martinez E, Gil GL, Benito AC, González-Billalabeitia E, Conesa MA, García García T, García-Garre E, Vicente V and Ayala de la Peña F: Tumor-infiltrating immune cell profiles and their change after neoadjuvant chemotherapy predict response and prognosis of breast cancer. Breast Cancer Res 16: 488, 2014

26. Zhang J and Guo Z: Immune escape of tumor. Chin J Cancer Biother 4: 315-317, 2006 (In Chinese)

27. Rettig L, Seidenberg S, Parvanova I, Samaras P, Curioni A, Knuth A and Pascolo S: Gemcitabine depletes regulatory T-cells in human and mice and enhances triggering of vaccine-specific cytotoxic T-cells. Int J Cancer 129: 832-838, 2011.
28. Maeda K, Hazama S, Tokuno K, Kan S, Maeda Y, Watanabe Y, Kamei R, Shindo Y, Maeda N, Yoshimura K, et al: Impact of chemotherapy for colorectal cancer on regulatory T-cells and tumor immunity. Anticancer Res 31: 4569-4574, 2011.

29. Van der Most RG, Currie AJ, Mahendran S, Prosser A, Darabi A, Robinson BW, Nowak AK and Lake RA: Tumor eradication after cyclophosphamide depends on concurrent depletion of regulatory T cells: A role for cycling TNFR2-expressing effector-suppressor $\mathrm{T}$ cells in limiting effective chemotherapy. Cancer Immunol Immunother 58: 1219-1228, 2009.

30. Frey DM, Droeser RA, Viehl CT, Zlobec I, Lugli A, Zingg U, Oertli D, Kettelhack C, Terracciano L and Tornillo L: High frequency of tumor-infiltrating FOXP3(+) regulatory T cells predicts improved survival in mismatch repair-proficient colorectal cancer patients. Int J Cancer 126: 2635-2643, 2010

31. Lin YC, Mahalingam J, Chiang JM, Su PJ, Chu YY, Lai HY, Fang JH, Huang CT, Chiu CT and Lin CY: Activated but not resting regulatory $\mathrm{T}$ cells accumulated in tumor microenvironment and correlated with tumor progression in patients with colorectal cancer. Int J Cancer 132: 1341-1350, 2013.

32. Llosa NJ, Cruise M, Tam A, Wicks EC, Hechenbleikner EM, Taube JM, Blosser RL, Fan H, Wang H, Luber BS, et al: The vigorous immune microenvironment of microsatellite instable colon cancer is balanced by multiple counter-inhibitory checkpoints. Cancer Discov 5: 43-51, 2015.

33. Saigusa S, Toiyama Y, Tanaka K, Inoue Y, Mori K, Ide S, Imaoka H, Kawamura M, Mohri Y and Kusunoki M: Implication of programmed cell death ligand 1 expression in tumor recurrence and prognosis in rectal cancer with neoadjuvant chemoradiotherapy. Int J Clin Oncol 21: 946-952, 2016.

34. Butte MJ, Keir ME, Phamduy TB, Sharpe AH and Freeman GJ: Programmed death-1 ligand 1 interacts specifically with the B7-1 costimulatory molecule to inhibit $\mathrm{T}$ cell responses. Immunity 27 $111-122,2007$.

35. Tamura H, Dong H, Zhu G, Sica GL, Flies DB, Tamada K and Chen L: B7-H1 costimulation preferentially enhances CD28-independent T-helper cell function. Blood 97: 1809-1816, 2001.

36. Parsa AT, Waldron JS, Panner A, Crane CA, Parney IF, Barry JJ, Cachola KE, Murray JC, Tihan T, Jensen MC, et al: Loss of tumor suppressor PTEN function increases B7-H1 expression and immunoresistance in glioma. Nat Med 13: 84-88, 2007.

37. Walker LS: Treg and CTLA-4: Two intertwining pathways to immune tolerance. J Autoimmun 45: 49-57, 2013.

38. Birebent B, Lorho R, Lechartier H, de Guibert S, Alizadeh M, $\mathrm{Vu}$ N, Beauplet A, Robillard N and Semana G: Suppressive properties of human $\mathrm{CD} 4+\mathrm{CD} 25+$ regulatory $\mathrm{T}$ cells are dependent on CTLA-4 expression. Eur J Immunol 34: 3485-3496, 2004

39. $\mathrm{Xu} \mathrm{C}, \mathrm{Li} \mathrm{W}$ and Yao Y: Regulating mechanism of regulatory $\mathrm{T}$ cells in immunoregulatory responses. Int J Pathol Clin Med 28: 199-204, 2008

40. Postow MA, Callahan MK, Barker CA, Yamada Y, Yuan J, Kitano S, Mu Z, Rasalan T, Adamow M, Ritter E, et al: Immunologic correlates of the abscopal effect in a patient with melanoma. N Engl J Med 366: 925-931, 2012

41. Qu Y, Zhang B, Liu S, Zhang A, Wu T and Zhao Y: 2-Gy whole-body irradiation significantly alters the balance of CD4+ CD25- T effector cells and CD4+ CD25+ Foxp3+ T regulatory cells in mice. Cell Mol Immunol 7: 419-427, 2010.

42. Billiard F, Buard V, Benderitter M and Linard C: Abdominal $\gamma$-radiation induces an accumulation of function-impaired regulatory $\mathrm{T}$ cells in the small intestine. Int J Radiat Oncol Biol Phys 80: 869-876, 2011.

43. Mizukami Y, Kono K, Kawaguchi Y, Akaike H, Kamimura K, Sugai H, Fujii H: Localisation pattern of Foxp3+ regulatory $\mathrm{T}$ cells is associated with clinical behaviour in gastric cancer. Br J Cancer 98: 148-153, 2008

44. Matsutani S, Shibutani M, Maeda K, Nagahara H, Fukuoka T, Nakao S, Hirakawa K and Ohira M: Significance of tumor-infiltrating lymphocytes before and after neoadjuvant therapy for rectal cancer. Cancer Sci 109: 966-979, 2018

This work is licensed under a Creative Commons Attribution-NonCommercial-NoDerivatives 4.0 International (CC BY-NC-ND 4.0) License. 